\title{
Body composition assessment and sarcopenia in patients with gastric cancer: a systematic review and meta-analysis
}

\author{
Sivesh K. Kamarajah ${ }^{1}\left[\right.$. James Bundred ${ }^{1}$ B Benjamin H. L. Tan ${ }^{1}$
}

Received: 5 July 2018 / Accepted: 20 September 2018 / Published online: 1 October 2018

(c) The International Gastric Cancer Association and The Japanese Gastric Cancer Association 2018

\begin{abstract}
Introduction There has recently been increased interest in the assessment of body composition in patients with gastric cancer for the purpose of prognostication. This systematic review and meta-analysis aim to evaluate the current literature on body composition assessment in patients with gastric cancer and its impact on peri-operative outcomes.

Methods A systematic literature search was conducted for studies reporting assessment of body composition in patients with gastric cancers. Meta-analysis of postoperative outcomes (overall and major complications, anastomotic leaks, pulmonary complications) and survival was performed using random effects models.

Results Thirty-nine studies reported the assessment of body composition in 8402 patients. Methods used to assess body composition in patients with gastric cancers were computerized tomography $(n=26)$, bioelectrical impedance analysis $(n=9)$, and dual-energy-X-ray-absorptiometry $(n=3)$. Only 21 studies reported the impact of pre-operative sarcopenia on post-operative outcomes. Sarcopenic patients have significantly higher rates of postoperative major complications $(n=12$, OR 1.67, $\left.\mathrm{CI}_{95 \%} 1.14-2.46, p=0.009\right)$, and pulmonary $\left(n=8\right.$, OR 4.01, $\left.\mathrm{CI}_{95 \%} 2.23-7.21, p<0.001\right)$ complications after gastrectomy. Meta-analysis of nine studies reporting overall survival after gastrectomy identified significantly worse survival in patients with pre-operative sarcopenia (HR 2.12, $\mathrm{CI}_{95 \%} 1.89-2.38, p<0.001$ ).

Conclusions Assessment of body composition has the potential to become a clinically useful tool that could support decisionmaking in patients with gastric cancer. However, variation in methods of assessing and reporting body composition in this patient group limits assessment of current post-operative outcomes
\end{abstract}

Keywords Gastric Cancer · Gastrectomy $\cdot$ Sarcopenia $\cdot$ Body composition · Outcomes · Survival

\section{Introduction}

Gastric cancers are the third leading cause of cancer death and the fifth most common cancer in the world, with half of all cases occurring in Eastern Asia, including Japan [1]. Solid tumours, including gastric cancers, are typically associated with a degree of anorexia as well as underlying metabolic alterations such as elevated energy expenditure, excess catabolism, and inflammation. In gastric cancers,

Electronic supplementary material The online version of this article (https://doi.org/10.1007/s10120-018-0882-2) contains supplementary material, which is available to authorized users.

Benjamin H. L. Tan

benjamin.tan@uhb.nhs.uk

1 Department of Upper Gastrointestinal Surgery, Queen Elizabeth Hospital Birmingham, Birmingham, UK these effects are worsened by the direct obstruction to the passage of food and combined effects of chemotherapy and major gastrectomy that results in a decline in nutritional intake [2, 3].

Recently, there has been growing interest relating to body composition assessment in patients diagnosed with gastric cancer for nutritional evaluation and prognostication. Measurement of skeletal muscle and/or adipose tissue have found success in a number of other areas of medicine for nutritional assessment [4-7], further supporting an argument for such measures to become part of clinical practice. There are, however, large variations in methods of assessment of body composition parameters. These include CT scans which are routinely used in clinical practice and have a high precision and specificity for muscle and fat distribution (visceral, intermuscular, and subcutaneous). Other measures less frequently used in cancer patients include dual-energy X-ray absorptiometry (DXA) and bioelectrical impedance (BIA). 
To further delineate the clinical utility of body composition assessment in patients with gastric cancers, it is considered timely that existing evidence are reviewed. This systematic review sought to summarise current literature surrounding body composition assessment in patients with gastric cancer and assess its impact on peri-operative outcomes and long-term survival.

\section{Methods}

\section{Search Strategy}

A systematic search of PubMed, EMBASE and the Cochrane Library databases were conducted on the 22nd June 2018 by two independent investigators. The search terms used were 'gastric cancer' or 'stomach cancer', and 'sarcopenia' or 'body composition' or 'muscular atrophy' individually or in combination. Search terms used for this review is presented as shown in Supplementary Table 1. The 'related articles' function was used to broaden the search, and all citations were considered for relevance. A manual search of reference lists in recent reviews and eligible studies was also undertaken. This paper is reported according to the PRISMA guidelines [8].

\section{Inclusion and exclusion criteria}

Inclusion criteria were: (1) studies reporting the assessment of body composition (by any method) in human subjects with gastric cancer (receiving palliative or curative treatment); (2) published in the English language. Exclusion criteria were: (1) Conference abstracts, review articles, and case reports ( $<5$ patients); (2) publications with mixed populations where the outcomes of patients with either benign disease or cancers at another site could not be separated from those of patients with gastric cancers. After excluding duplicates, two researchers (SKK, BHT) independently reviewed the titles and abstracts of studies identified by the literature search. Where a study was considered to be potentially relevant to the research question a full copy of the publication was obtained for further review. The reference lists of all included studies were hand-searched in order to identify other potentially relevant studies. Any areas of disagreement between the two primary researchers were resolved through discussion.

\section{Study outcomes}

The primary outcome measure was post-operative complications such as overall complications (Grade I-V) and major complications ( $\geq$ Grade III) reported according to Clavien-Dindo Classification [9]. Secondary outcome measures were survival (overall, recurrence-free and cancer-specific) and surgery-specific complications (pulmonary, cardiac, surgical site infections).

\section{Data extraction}

One researcher (SKK) extracted data on study characteristics (author, year of publication, country of origin, study design, patient number), patient demographics (age, sex, AJCC T Classification, AJCC Stage), method and details of body and reported clinical outcomes. Body composition measures were abstracted according to method of assessment (CT, DXA, BIA), definition and cut-off points defining sarcopenia and obesity.

\section{Definitions}

Gastric cancer is a malignancy of any portion of the stomach. Body composition assessment is any method of reporting either the volume or characteristics of muscle and/or adipose compartments within the body. Sarcopenia is defined as severe depletion of skeletal muscle mass that has been defined by a range of criteria that are specific for the method of assessment. Cachexia is a multifactorial syndrome characterized by ongoing loss of skeletal muscle mass (with or without loss of fat), that is not fully reversible using conventional nutritional support and that eventually leads to functional impairment $[10,11]$.

\section{Assessment of methodological quality}

Methodological quality and standard of outcome reporting within included studies were assessed by two independent researchers (SKK, BT). Methodological quality was formally assessed using the Newcastle-Ottawa score for cohort studies (SKK, JB).

\section{Statistical analysis}

This systematic review and meta-analysis were conducted in accordance with the recommendations of the Cochrane Library and MOOSE guidelines [12]. For categorical variables, analysis was performed by calculating the odds ratio (OR). For survival data, analysis was performed by calculating the logarithm of the hazard ratio (HR) with $95 \%$ confidence intervals $\left(\mathrm{CI}_{95 \%}\right)$. HR and its variance were extracted directly from the published manuscript. Where these data were not available it was determined through additional calculations that were dependent on the data presented by the study: annual mortality rates, survival curves, number of deaths, or percentage freedom from death [13]. The random effects, the DerSimonian-Laird method was used for the meta-analysis of outcomes. Funnel plots were used to 
visually assess publication bias of included studies. Heterogeneity between studies was assessed using the $I^{2}$ value to determine the degree of variation not attributable to chance alone. $I^{2}$ values were considered to represent low, moderate, and high degrees of heterogeneity where values were $<25 \%$, $25-75 \%$, and $>75 \%$, respectively. Funnel plot asymmetry was assessed using the Egger test. Statistical significance was considered when $p<0.05$. Statistical analyses were performed using the RevMan 5.3 software (Copenhagen: The Nordic Cochrane Centre, The Cochrane Collaboration, 2011) and Stata 15 (Version 15.1, StataCorp, College Station, Texas).

\section{Results}

\section{Study Characteristics}

The literature search identified 39 studies reported according to the PRISMA guidelines as shown in Fig. 1. Majority of studies identified were from Asia $(n=33)$ [14-46] followed by Europe $(n=5)[5,47-50]$, and North America $(n=1)$ [51].

\section{Reporting standards and methodological quality}

Criteria for defining parameters of body composition varied considerably (Table 1). CT cut-points defining sarcopenia were reported in 21 studies, all of which adopting sex specific cut-off points. Of the 21 studies, only 18 studies reported normalisation of these measurements to height $(n=17)$ and body surface area $(n=1)[5,17,19,21,23$, $29,33,34,37,38,40,41,43-45,49,51]$. Studies were also heterogeneous with respect to several factors known to influence body composition: race, sex, BMI, and tumour characteristics (Table 2). Variation in these factors was broadly reflective of known differences that exist between Asian and Western populations. Study quality was assessed using NOS and a breakdown of each components are presented under Supplementary Table 2. A summary of studies reporting
Fig. 1 Flow diagram of included studies

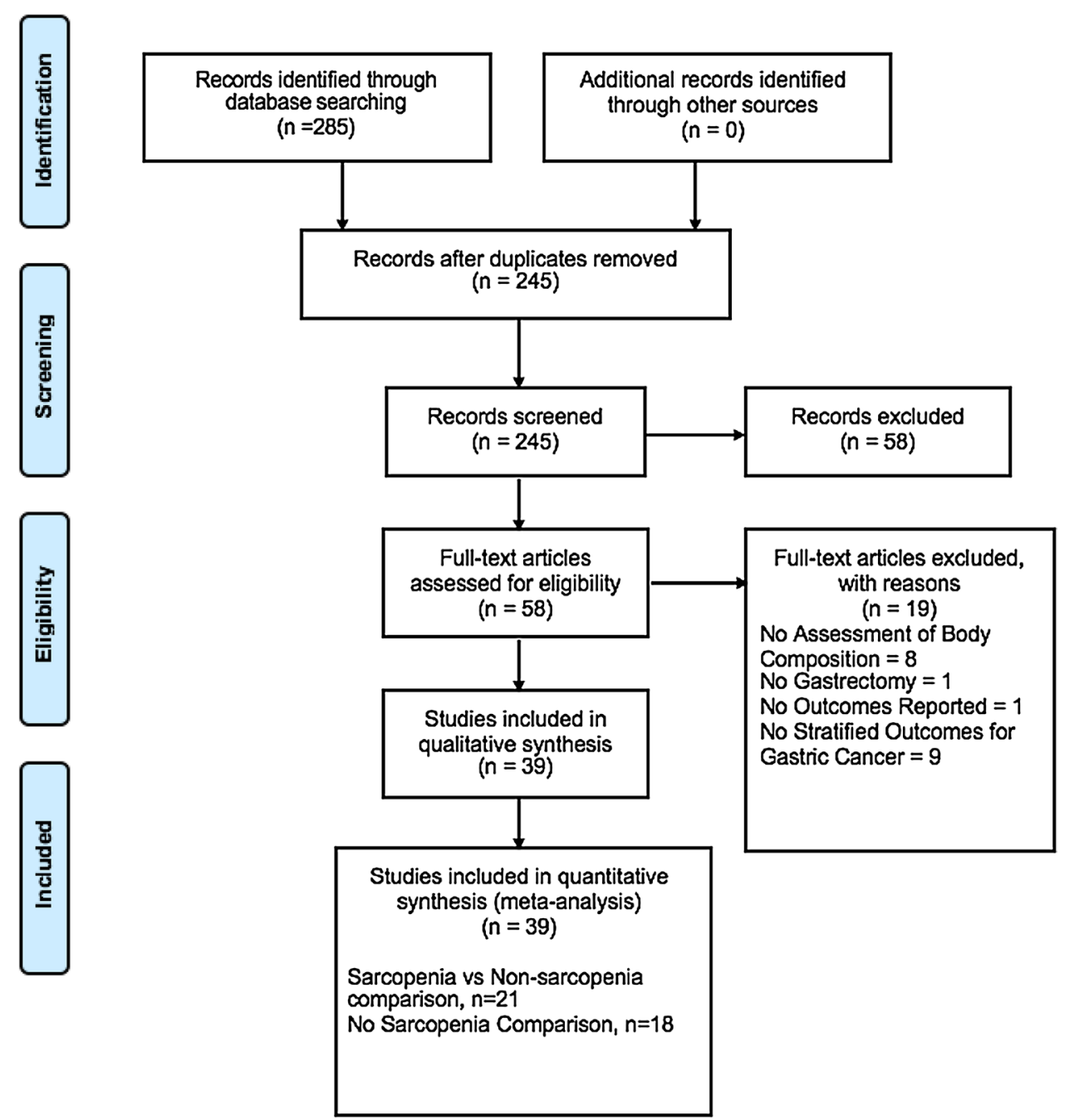


Table 1 Details of body composition assessment methodology

\begin{tabular}{|c|c|c|c|c|c|c|c|c|c|}
\hline Authors & Year & Region & Method & $\begin{array}{l}\text { Time of } \\
\text { assessment }\end{array}$ & $\begin{array}{l}\text { CT level of } \\
\text { assessment }\end{array}$ & CT muscle & $\begin{array}{l}\text { CT adipose } \\
\text { tissue }\end{array}$ & $\begin{array}{l}\text { Normalisa- } \\
\text { tion of meas- } \\
\text { urements }\end{array}$ & Definition \\
\hline Kiyama [27] & 2005 & Asia & BIA & PreOp, PO & - & - & - & No & - \\
\hline Aoyama [14] & 2015 & Asia & BIA & PreOp, PO & - & - & - & No & - \\
\hline Fukuda [20] & 2015 & Asia & BIA & PreOp & - & - & - & No & $\begin{array}{l}8.87 \mathrm{~kg} / \mathrm{m}^{2} \\
\quad(\mathrm{men}), 6.42 \mathrm{~kg} / \\
\mathrm{m}^{2} \text { (women) }\end{array}$ \\
\hline $\begin{array}{l}\text { Kobayashi } \\
\text { [30] }\end{array}$ & 2015 & Asia & BIA & PreOp, PO & - & - & - & No & - \\
\hline Aoyama [17] & 2016 & Asia & BIA & PreOp, PO & - & - & - & No & - \\
\hline Sato [41] & 2016 & Asia & BIA & PreOp & - & - & - & No & $\begin{array}{l}7.25 \mathrm{~kg} / \mathrm{m}^{2} \\
(\mathrm{men}), 5.67 \mathrm{~kg} / \\
\mathrm{m}^{2} \text { (women) }\end{array}$ \\
\hline $\begin{array}{l}\text { Yamamoto } \\
\text { [43] }\end{array}$ & 2017 & Asia & BIA & $\begin{array}{l}\text { Pre-Tx, Post- } \\
\text { Tx }\end{array}$ & - & - & - & Yes $(\mathrm{H})$ & $\begin{array}{l}43 \mathrm{~cm}^{2} / \mathrm{m}^{2} \\
\text { (men with } \\
\mathrm{BMI}<25.0 \mathrm{~kg} / \\
\left.\mathrm{m}^{2}\right), 53 \mathrm{~cm}^{2} / \\
\mathrm{m}^{2}(\text { men with } \\
\mathrm{BMI}>25 \mathrm{~kg} / \\
\left.\mathrm{m}^{2}\right), 41 \mathrm{~cm}^{2} / \mathrm{m}^{2} \\
\text { (women) }\end{array}$ \\
\hline Aoyama [18] & 2018 & Asia & BIA & PreOp, PO & - & - & - & No & - \\
\hline Correia [48] & 2007 & Europe & BIA & PreOp & - & - & - & No & - \\
\hline Hiki [22] & 2009 & Asia & $\mathrm{CT}$ & PreOp & - & - & - & No & - \\
\hline Jeong [26] & 2009 & Asia & $\mathrm{CT}$ & PreOp & - & - & - & No & - \\
\hline Kunisaki [32] & 2011 & Asia & $\mathrm{CT}$ & PreOp & - & - & - & No & - \\
\hline Miyaki [37] & 2013 & Asia & $\mathrm{CT}$ & PreOp & - & - & - & No & - \\
\hline Kim [28] & 2014 & Asia & $\mathrm{CT}$ & PreOp & $\mathrm{T} 10$ & - & $\begin{array}{l}\text { (-) } 190 \text { to } \\
-30\end{array}$ & No & - \\
\hline Yamaoka [44] & 2015 & Asia & $\mathrm{CT}$ & PreOp, PO & L3 mid-point & (-) 30 to 110 & $\begin{array}{l}\text { (-) } 190 \text { to } \\
-30\end{array}$ & No & - \\
\hline Chen [19] & 2016 & Asia & $\mathrm{CT}$ & PreOp & L3 mid-point & (-) 29 to 150 & - & Yes $(\mathrm{H})$ & $\begin{array}{l}<40.8 \mathrm{~cm}^{2} / \\
\mathrm{m}^{2}(\mathrm{men}), \\
<34.9 \mathrm{~cm}^{2} / \mathrm{m}^{2} \\
\text { (women) }\end{array}$ \\
\hline Hayashi [21] & 2016 & Asia & CT & PreTx & L3 mid-point & $(-) 29$ to 150 & - & Yes $(\mathrm{H})$ & $\begin{array}{l}<43 \mathrm{~cm}^{2} / \\
\mathrm{m}^{2}(\mathrm{men}) \\
<41 \mathrm{~cm}^{2} / \mathrm{m}^{2} \\
\text { (women) }\end{array}$ \\
\hline Huang [23] & 2016 & Asia & $\mathrm{CT}$ & PreOp & L3 mid-point & $(-) 29$ to 150 & - & Yes $(\mathrm{H})$ & $\begin{array}{l}<40.8 \mathrm{~cm}^{2} / \\
\mathrm{m}^{2}(\mathrm{men}), \\
<34.9 \mathrm{~cm}^{2} / \mathrm{m}^{2} \\
\text { (women) }\end{array}$ \\
\hline Nishigori [39] & 2016 & Asia & CT & PreOp & L3 mid-point & $(-) 29$ to 150 & $\begin{array}{l}\text { (-) } 190 \text { to } \\
-30\end{array}$ & Yes $(\mathrm{H})$ & $\begin{array}{l}\leq 52.4 \mathrm{~cm}^{2} / \\
\mathrm{m}^{2}(\mathrm{men}), \\
\leq 38.5 \mathrm{~cm}^{2} / \mathrm{m}^{2} \\
\text { (women) }\end{array}$ \\
\hline Wang [42] & 2016 & Asia & $\mathrm{CT}$ & PreOp & L3 mid-point & $(-) 29$ to 150 & - & Yes $(\mathrm{H})$ & $\begin{array}{l}<36.0 \mathrm{~cm}^{2} / \\
\mathrm{m}^{2}(\mathrm{men}), \\
<29.0 \mathrm{~cm}^{2} / \mathrm{m}^{2} \\
\text { (women) }\end{array}$ \\
\hline Zhuang [1] & 2016 & Asia & CT & PreOp & L3 mid-point & $(-) 29$ to 150 & - & Yes $(\mathrm{H})$ & $\begin{array}{l}<40.8 \mathrm{~cm}^{2} / \\
\mathrm{m}^{2}(\mathrm{men}), \\
<34.9 \mathrm{~cm}^{2} / \mathrm{m}^{2} \\
\text { (women) }\end{array}$ \\
\hline
\end{tabular}


Table 1 (continued)

\begin{tabular}{|c|c|c|c|c|c|c|c|c|c|}
\hline Authors & Year & Region & Method & $\begin{array}{l}\text { Time of } \\
\text { assessment }\end{array}$ & $\begin{array}{l}\text { CT level of } \\
\text { assessment }\end{array}$ & CT muscle & $\begin{array}{l}\text { CT adipose } \\
\text { tissue }\end{array}$ & $\begin{array}{l}\text { Normalisa- } \\
\text { tion of meas- } \\
\text { urements }\end{array}$ & Definition \\
\hline Huang [25] & 2017 & Asia & $\mathrm{CT}$ & PreOp & L3 mid-point & (-) 29 to 150 & $\begin{array}{l}\text { (-) } 150 \text { to } \\
-50\end{array}$ & Yes $(\mathrm{H})$ & $\begin{array}{l}<40.8 \mathrm{~cm}^{2} / \\
\mathrm{m}^{2}(\mathrm{men}), \\
<34.9 \mathrm{~cm}^{2} / \mathrm{m}^{2} \\
\text { (women) }\end{array}$ \\
\hline Huang [24] & 2017 & Asia & $\mathrm{CT}$ & PreOp, PO & L3 mid-point & (-) 29 to 150 & - & Yes $(\mathrm{H})$ & - \\
\hline Kudou [31] & 2017 & Asia & $\mathrm{CT}$ & PreOp & L3 mid-point & (-) 29 to 150 & - & Yes $(\mathrm{H})$ & $\begin{array}{l}43 \mathrm{~cm}^{2} / \mathrm{m}^{2} \\
\text { (men with } \\
\mathrm{BMI}<25.0 \mathrm{~kg} / \\
\left.\mathrm{m}^{2}\right), 53 \mathrm{~cm}^{2} / \\
\mathrm{m}^{2}(\text { men with } \\
\mathrm{BMI}>25 \mathrm{~kg} / \\
\left.\mathrm{m}^{2}\right), 41 \mathrm{~cm}^{2} / \mathrm{m}^{2} \\
\text { (women) }\end{array}$ \\
\hline Lou [35] & 2017 & Asia & $\mathrm{CT}$ & PreOp & L3 mid-point & (-) 29 to 150 & - & Yes $(\mathrm{H})$ & $\begin{array}{l}<40.8 \mathrm{~cm}^{2} / \\
\mathrm{m}^{2}(\mathrm{men}) \\
<34.9 \mathrm{~cm}^{2} / \mathrm{m}^{2} \\
\text { (women) }\end{array}$ \\
\hline Nagata [38] & 2017 & Asia & $\mathrm{CT}$ & PreOp & L3 mid-point & - & - & Yes $(\mathrm{H})$ & - \\
\hline Sakurai [40] & 2017 & Asia & $\mathrm{CT}$ & PreOp & L3 mid-point & (-) 29 to 150 & - & Yes $(\mathrm{H})$ & $\begin{array}{l}<43.2 \mathrm{~cm}^{2} / \\
\mathrm{m}^{2}(\mathrm{men}) \\
<34.6 \mathrm{~cm}^{2} / \mathrm{m}^{2} \\
\text { (women) }\end{array}$ \\
\hline Zheng [45] & 2017 & Asia & $\mathrm{CT}$ & PreOp & L3 mid-point & (-) 29 to 150 & - & Yes $(\mathrm{H})$ & $\begin{array}{l}<32.5 \mathrm{~cm}^{2} / \\
\mathrm{m}^{2}(\mathrm{men}) \\
<28.6 \mathrm{~cm}^{2} / \mathrm{m}^{2} \\
\text { (women) }\end{array}$ \\
\hline Zhou [46] & 2017 & Asia & CT & PreOp & L3 mid-point & (-) 29 to 150 & - & Yes $(\mathrm{H})$ & $\begin{array}{l}<40.8 \mathrm{~cm}^{2} / \\
\mathrm{m}^{2}(\mathrm{men}), \\
<34.9 \mathrm{~cm}^{2} / \mathrm{m}^{2} \\
\text { (women) }\end{array}$ \\
\hline $\begin{array}{l}\text { Kugimiya } \\
\text { [47] }\end{array}$ & 2018 & Asia & $\mathrm{CT}$ & PreOp, PO & L3 mid-point & $(-) 30$ to 110 & - & Yes $(\mathrm{H})$ & - \\
\hline Kuwada [33] & 2018 & Asia & $\mathrm{CT}$ & PreOp & L3 mid-point & $(-) 30$ to 150 & - & Yes (BSA) & $\begin{array}{l}69.7 \mathrm{~cm}^{2} / \\
\mathrm{m}^{2}(\mathrm{men}) \\
54.2 \mathrm{~cm}^{2} / \mathrm{m}^{2} \\
\text { (women) }\end{array}$ \\
\hline Lu [36] & 2018 & Asia & CT & PreOp & L3 mid-point & $(-) 30$ to 110 & - & Yes $(\mathrm{H})$ & $\begin{array}{l}512.7 \mathrm{~mm} 2 / \\
\mathrm{m} 2(\mathrm{men}), \\
344.3 \mathrm{~mm} 2 / \mathrm{m} 2 \\
\text { (women) }\end{array}$ \\
\hline Tegels [50] & 2015 & Europe & CT & PreOp & L3 mid-point & $(-) 30$ to 110 & $\begin{array}{l}(-) 190 \text { to } \\
-30\end{array}$ & Yes $(\mathrm{H})$ & $\begin{array}{l}43 \mathrm{~cm}^{2} / \mathrm{m}^{2} \\
\text { (men with } \\
\mathrm{BMI}<25.0 \mathrm{~kg} / \\
\left.\mathrm{m}^{2}\right), 53 \mathrm{~cm}^{2} / \\
\mathrm{m}^{2}(\text { men with } \\
\mathrm{BMI}>25 \mathrm{~kg} / \\
\left.\mathrm{m}^{2}\right), 41 \mathrm{~cm}^{2} / \mathrm{m}^{2} \\
\text { (women) }\end{array}$ \\
\hline Palmela [7] & 2017 & Europe & $\mathrm{CT}$ & PreOp & L3 mid-point & (-) 29 to 150 & - & Yes $(\mathrm{H})$ & $\begin{array}{l}43 \mathrm{~cm}^{2} / \mathrm{m}^{2} \\
\text { (men with } \\
\mathrm{BMI}<25.0 \mathrm{~kg} / \\
\left.\mathrm{m}^{2}\right), 53 \mathrm{~cm}^{2} / \\
\mathrm{m}^{2}(\text { men with } \\
\mathrm{BMI}>25 \mathrm{~kg} / \\
\left.\mathrm{m}^{2}\right), 41 \mathrm{~cm}^{2} / \mathrm{m}^{2} \\
\text { (women) }\end{array}$ \\
\hline
\end{tabular}


Table 1 (continued)

\begin{tabular}{|c|c|c|c|c|c|c|c|c|c|}
\hline Authors & Year & Region & Method & $\begin{array}{l}\text { Time of } \\
\text { assessment }\end{array}$ & $\begin{array}{l}\text { CT level of } \\
\text { assessment }\end{array}$ & CT muscle & $\begin{array}{l}\text { CT adipose } \\
\text { tissue }\end{array}$ & $\begin{array}{l}\text { Normalisa- } \\
\text { tion of meas- } \\
\text { urements }\end{array}$ & Definition \\
\hline Mirkin [52] & 2017 & $\begin{array}{l}\text { North } \\
\text { America }\end{array}$ & $\mathrm{CT}$ & $\begin{array}{l}\text { Pre-Tx, Post- } \\
\text { Tx }\end{array}$ & L3 mid-point & - & - & Yes $(\mathrm{H})$ & $\begin{array}{l}<545 \mathrm{~mm}^{2} / \\
\mathrm{m}^{2}(\mathrm{men}), \\
<385 \mathrm{~mm}^{2} / \mathrm{m}^{2} \\
\text { (women) }\end{array}$ \\
\hline Lee [34] & 2018 & Asia & DXA & PreOp, PO & - & - & - & No & - \\
\hline Liedman [49] & 1998 & Europe & DXA & PreOp, PO & - & - & - & No & - \\
\hline Copland [51] & 2007 & Europe & DXA & PreOp, PO & - & - & - & No & - \\
\hline $\begin{array}{l}\text { Kawamura } \\
\text { [27] }\end{array}$ & 2018 & Asia & TSF & PreOp & - & - & - & No & $\begin{array}{l}<38.05 \mathrm{~cm}^{2} \\
\quad(\text { men) }, \\
<27.87 \mathrm{~cm}^{2} \\
\text { (women) }\end{array}$ \\
\hline
\end{tabular}

CT muscle and adipose tissue reported as Hounsfield unit threshold

$B I A$ body impedance analysis, $B S A$ adjusted for body surface area, $C T$ computer tomography, $D X A$ dual-energy X-ray absorptiometry, $H$ adjusted for height, $P O$ post-operative, Post-Tx post-treatment (including neo-adjuvant therapy), PreOp pre-operative, Pre-Tx pre-treatment (including neo-adjuvant therapy), TSF thickness of skin fold

impact of pre-operative sarcopenia on morbidity and mortality as Supplementary Table 3.

\section{Body composition assessment in gastric cancer}

Methods of assessment used in each study, including criteria for key descriptors of body composition are presented in Table 1. CT ( $n=26$ studies) [5, 17, 19-24, 26, 29-31, 33-38, $40,42-46,49,51]$ and BIA $(n=9)$ [14-16, 18, 27, 28, 39, $41,47]$ were the most commonly reported methods of body composition assessment, while DXA was utilized in three studies [32, 48, 50, 52]. Of the 25 studies published since January 1, 2016, CT was the chosen method of assessment in $19(76 \%)$. A total of 3193 patients were evaluated (CT, $n=5999$; BIA, $n=1366$; DXA, $n=86$ ) (Table 2). Assessment was predominantly performed in patients with resectable gastric cancer prior to surgery either before and/or after neoadjuvant therapy. The reported prevalence of sarcopenia in preoperative gastric cancer patients in 21 studies varied between $7 \%$ and $70 \%$. Prevalence values can only be compared between studies that used identical criteria for sarcopenia in populations with similar characteristics; other comparisons are unreliable owing to differences in methodologies, patient populations, and criteria for defining this parameter (Tables 1, 2).

\section{Postoperative morbidity and mortality}

Impact of preoperative sarcopenia on overall complications was reported in 14 studies [18, 23, 25, 29, 31, 33, 34, 38-41, $44,45,51]$. Preoperative sarcopenia was associated with significantly higher rates of overall postoperative complications (33\% vs 23\%, OR 2.18, $\mathrm{CI}_{95 \%} 1.49-3.20, p<0.001, I^{2}$
$81 \%$ ) (Fig. 2a). Impact of preoperative sarcopenia on major complications was reported in 13 studies [17, 18, 23, 29, 31, 33, 34, 38, 40, 41, 44, 45, 49]. Preoperative sarcopenia was associated with significantly higher rates of major postoperative complications ( $13 \%$ vs $7 \%$, OR $1.67, \mathrm{CI}_{95 \%}$ $1.14-2.46, p=0.009, I^{2} 53 \%$ ) (Fig. 2b). Impact of preoperative sarcopenia on pulmonary complications was reported in 9 studies [18, 23, 25, 29, 33, 39-41, 44, 52]. Preoperative sarcopenia was associated with significantly higher rates of postoperative pulmonary complications (13\% vs $4 \%$, OR 4.01, $\mathrm{CI}_{95 \%}$ 2.23-7.21, $p<0.001, I^{2} 47 \%$ ) (Fig. 2c). Impact of preoperative sarcopenia on cardiac complications was reported in 7 studies [18, 23, 25, 33, 40, 41, 44]. Preoperative sarcopenia was associated with significantly higher rates of postoperative cardiac complications (4\% vs $2 \%$, OR 4.11, $\mathrm{CI}_{95 \%}$ 1.54-11.02, $p=0.005, I^{2} 26 \%$ ) (Fig. 2d).

Impact of preoperative sarcopenia on mortality was reported in ten studies $[18,23,25,29,33,38,40,44,45$, 49]. Preoperative sarcopenia was associated with significantly higher rates of mortality ( $2 \%$ vs $1 \%$, OR $2.17, \mathrm{CI}_{95 \%}$ 1.06-4.43, $p=0.03, I^{2} 0 \%$ ) (Fig. 2e). Egger regression testing suggested publication biases were minimal for reporting of overall complications $(p=0.219)$, major complications $(p=0.655)$, pulmonary complications $(p=0.503)$, and cardiac complications $(p=0.220)$ and mortality $(p=0.612)$ (Supplementary Fig. 1).

There was no increased risk of non-pulmonary postoperative complications in patients with sarcopenia, including: anastomotic leak (nine studies: OR 1.09, $\mathrm{CI}_{95 \%}$ 0.62-1.94, $p=0.76, I^{2} 0 \%$ ) (Supplementary Fig. 2) [18, 23, 25, 29, 33, 37, 39, 40, 44]; surgical site infections (nine studies: OR 0.97, $\mathrm{CI}_{95 \%}$ 0.69-1.37, $p=0.87, I^{2} 0 \%$ ) (Supplementary Fig. 3) [18, 23, 25, 33, 37, 38, 40, 41, 44]. These 


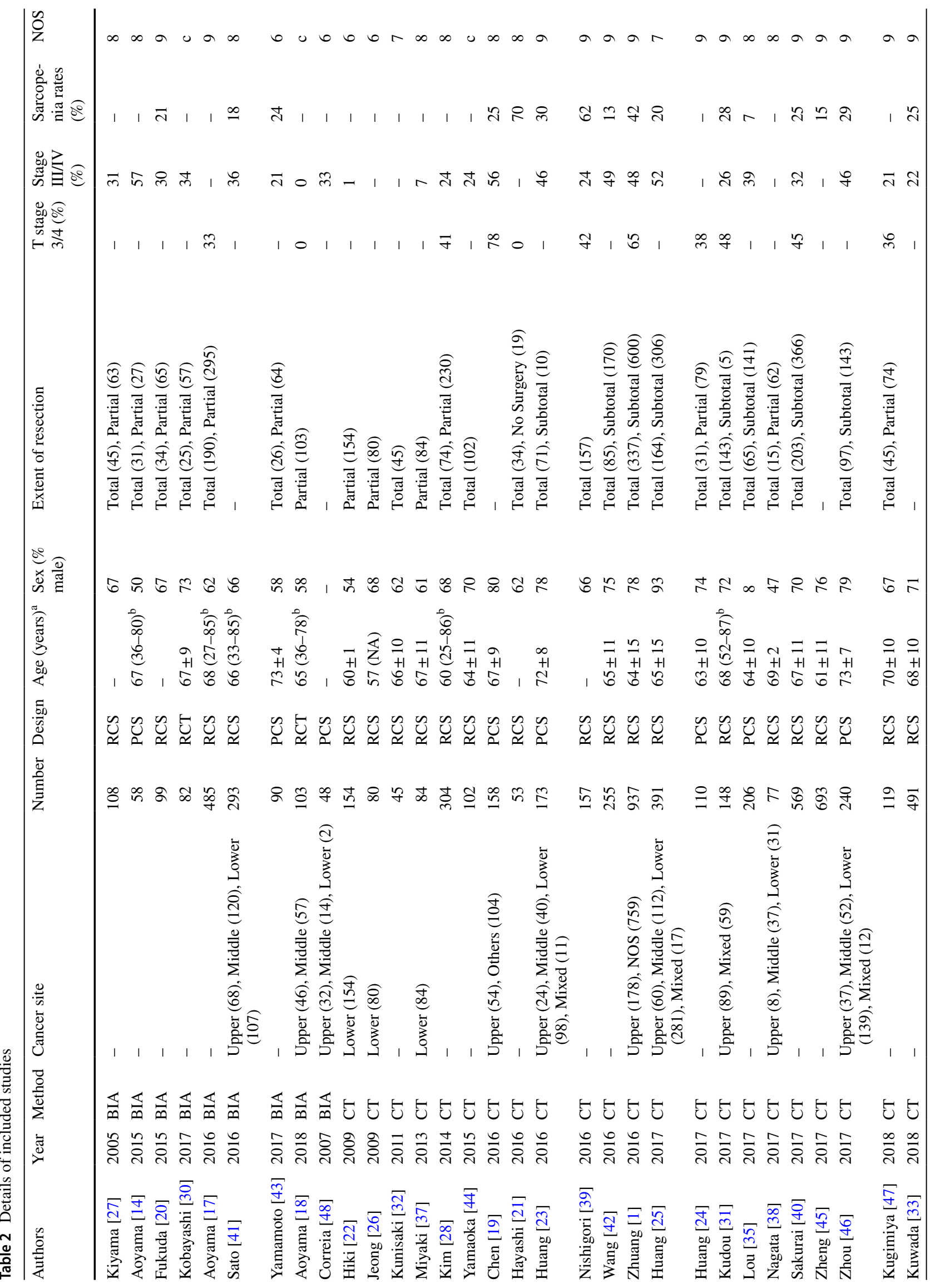




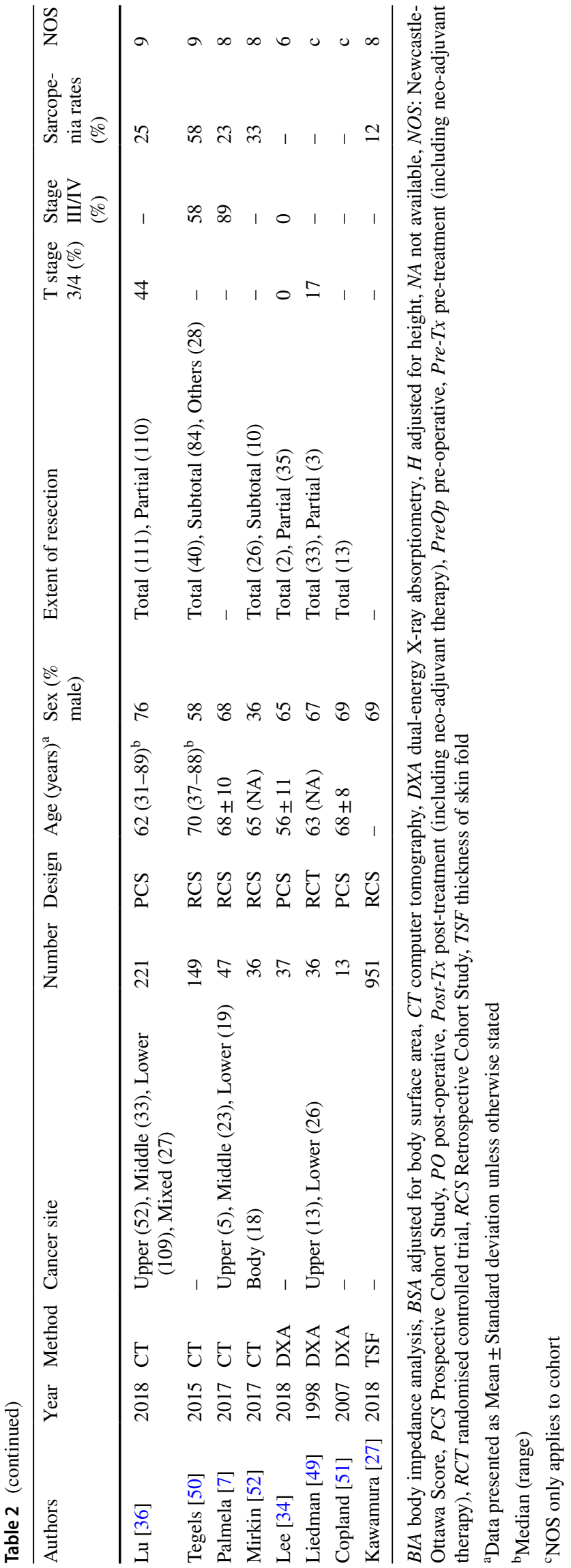

Fig. 2 Summary meta-analysis of studies reporting impact of preoperative sarcopenia on post-operative outcomes in patients undergoing gastrectomy. a Overall, b major, c pulmonary complications, d cardiac complications, $\mathbf{e}$ in-hospital mortality

relationships remained non-significant following reanalysis of only those studies utilizing CT for the assessment of sarcopenia. Egger regression testing suggested publication biases were minimal for reporting of anastomotic leak $(p=0.514)$ and surgical site infections $(p=0.409)$ (Supplementary Fig. 1).

\section{Long-term survival}

The influence of preoperative sarcopenia on the overall survival of patients following treatment for gastric cancer was reported in nine studies [19, 21, 25, 29, 31, 34, 38, 43, 45]. Meta-analysis of outcomes presented within these studies identified that sarcopenia was associated with lower overall survival (HR 2.12, $\mathrm{CI}_{95 \%}$ 1.89-2.38, $p<0.001$ ) (Fig. 3a). Heterogeneity between studies was moderate $\left(I^{2} 37 \%\right)$. The influence of preoperative sarcopenia on the recurrence-free $[34,43,45]$ and cancer-specific survival $[25,34,38]$ of patients following treatment for gastric cancer was reported in three studies respectively. Meta-analysis of outcomes presented within these studies identified that sarcopenia was associated with lower recurrence-free survival (HR 2.12, $\mathrm{CI}_{95 \%}$ 1.82-2.47, $p<0.001, I^{2} 40 \%$ ) (Fig. 3b) and cancerspecific survival (HR 2.00, $\mathrm{CI}_{95 \%} 1.54-2.59, p<0.001, I^{2}$ $0 \%$ ) (Fig. 3c). Egger regression testing suggested publication biases were minimal for reporting of overall survival ( $p=0.315)$, recurrence free survival $(p=0.716)$ and cancer specific survival $(p=0.800)$ (Supplementary Fig. 1).

\section{Sensitivity analysis on adjusted outcomes}

Sensitivity analysis of studies reporting multivariable analysis on the influence of pre-operative sarcopenia on overall complications, major complications and overall survival were performed (Supplementary Fig. 4). Three studies reported adjusted odds ratio for overall [17, 21, 44] and major [18, 40,45] complications. Meta-analysis of odds ratios derived from multivariable analysis found pre-operative sarcopenia to be independently associated with poorer overall complications (HR 2.50, $\mathrm{CI}_{95 \%} 1.68-3.72 ; p<0.001$, $I^{2} 3 \%$; Fig. 3a) and major complications (HR 3.62, $\mathrm{CI}_{95 \%}$ $2.34-5.59 ; p<0.001, I^{2} 0 \%$, Fig. 3b).

Seven studies reported adjusted hazard ratios for overall survival [14, 19, 25, 29, 31, 34, 38, 43, 45]. Meta-analysis of hazard ratios derived from multivariable analysis found pre-operative sarcopenia to be independently associated with poorer overall survival (HR 1.76, $\mathrm{CI}_{95 \%} 1.53-2.03$; 


\begin{tabular}{|c|c|c|c|c|c|c|c|}
\hline & Sarcop & enia & Non-Sarc & enia & & Odds Ratio & \\
\hline Study or Subgroup & Events & Total & Events & Total & Weight & $\mathrm{M}-\mathrm{H}$, Random, $95 \% \mathrm{CI}$ & Year \\
\hline Fukuda 2015 & 12 & 21 & 28 & 78 & $6.3 \%$ & $2.38[0.89,6.35]$ & 2015 \\
\hline Sato 2016 & 12 & 54 & 27 & 239 & $7.5 \%$ & $2.24[1.05,4.78]$ & 2016 \\
\hline Wang 2016 & 14 & 32 & 32 & 223 & $7.3 \%$ & $4.64[2.10,10.25]$ & 2016 \\
\hline Zhuang 2016 & 111 & 389 & 116 & 548 & $10.0 \%$ & $1.49[1.10,2.01]$ & 2016 \\
\hline Huang 2017 & 35 & 79 & 36 & 391 & $8.7 \%$ & $7.84[4.48,13.74]$ & 2017 \\
\hline Kudou 2017 & 10 & 42 & 33 & 106 & $7.1 \%$ & $0.69[0.30,1.57]$ & 2017 \\
\hline Laou 2017 & 9 & 14 & 46 & 192 & $5.5 \%$ & $5.71[1.82,17.91]$ & 2017 \\
\hline Mirkin 2017 & 6 & 12 & 6 & 24 & $4.2 \%$ & $3.00[0.70,12.93]$ & 2017 \\
\hline Sakurai 2017 & 33 & 142 & 121 & 427 & $9.3 \%$ & $0.77[0.49,1.19]$ & 2017 \\
\hline Zhou 2017 & 34 & 69 & 42 & 171 & $8.5 \%$ & $2.98[1.66,5.36]$ & 2017 \\
\hline Lu 2018 & 10 & 55 & 16 & 166 & $6.9 \%$ & $2.08[0.88,4.91]$ & 2018 \\
\hline Kawamura 2018 & 56 & 111 & 286 & 840 & $9.5 \%$ & $1.97[1.32,2.94]$ & 2018 \\
\hline Kuwada 2018 & 36 & 123 & 80 & 368 & $9.2 \%$ & $1.49[0.94,2.36]$ & 2018 \\
\hline Total $(95 \% \mathrm{Cl})$ & & 1143 & & 3773 & $100.0 \%$ & $2.18[1.49,3.20]$ & \\
\hline Total events & 378 & & 869 & & & & \\
\hline
\end{tabular}

Heterogeneity. $\mathrm{Tau}^{2}=0.36 ; \mathrm{Chi}^{2}=61.93, \mathrm{df}=12(\mathrm{P}<0.00001) ; \mathrm{I}^{2}=81 \%$

Test for overall effect: $z=3.99(\mathrm{P}<0.0001)$

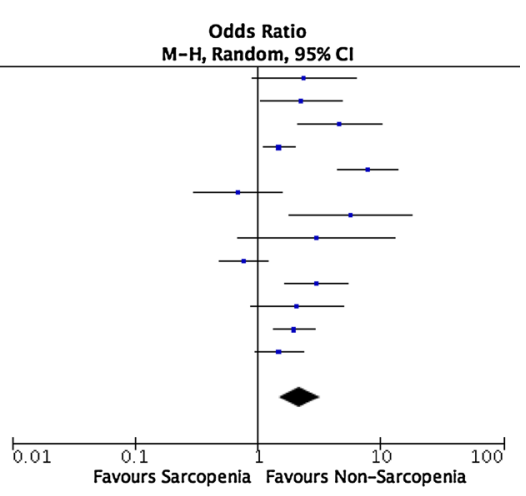

B

Sarcopenia Non-Sarcopenia

Odds Ratio

\begin{tabular}{cccccc} 
Study or Subgroup & \multicolumn{2}{c}{ Sarcopenia } & \multicolumn{2}{c}{ Non-Sarcopenia } & Odds Ratio \\
Events Total & Events & Total Weight $\mathrm{M}-\mathrm{H}$, Random, $95 \% \mathrm{Cl}$ Year
\end{tabular}

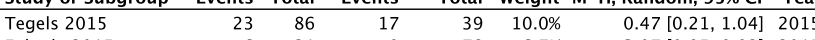

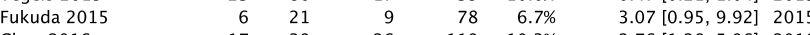

Chen 2016

Wang 2016

Zhuang 2016

$17 \quad 39$

Shuakurai 2017

Zhou 2017

Huang 2017

Kudou 2017

Laou 2017

Kuwada 2018

Lu 2018

\begin{tabular}{rrrrr}
17 & 39 & 26 & 119 & $10.3 \%$ \\
\hline & 32 & 6 & 223 & $5.1 \%$
\end{tabular}

$\begin{array}{rrrrr}40 & 389 & 21 & 548 & 12.8 \%\end{array}$

$\begin{array}{lllll}15 & 142 & 49 & 427 & 12.0 \%\end{array}$

$\begin{array}{rrrrr}6 & 69 & 10 & 171 & 7.6 \% \\ 7 & 79 & 16 & 391 & 8.7 \%\end{array}$

$\begin{array}{lllll}7 & 79 & 16 & 391 & 8.7 \% \\ 4 & 42 & 11 & 106 & 6.5 \%\end{array}$

$\begin{array}{lllll}4 & 42 & 11 & 106 & 6.5 \% \\ 2 & 14 & 11 & 192 & 4.3 \%\end{array}$

$2.76[1.28,5.96] 2016$
$3.74[0.89,15.78]$
2016

$3.74[0.89,15.78] 2016$
$2.88[1.67,4.96]$

$2.88[1.67,4.96] 2016$

$0.91[0.49,1.68] 2017$

$1.53[0.53,4.40] 2017$

$2.28[0.91,5.74] 2017$

$0.91[0.27,3.03] 2017$

$2.74[0.54,13.80] 2017$

$1.40[0.72,2.72] 2018$

Total $(95 \% \mathrm{Cl})$

$\begin{array}{rr}14 & 123 \\ 3 & 55\end{array}$

$31 \quad 368 \quad 11.4 \%$

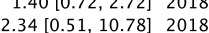

Total $(95 \% \mathrm{C})$
Total events

1091

$2828 \quad 100.0 \%$

$1.67[1.14,2.46]$

Heterogeneity: $\mathrm{Tau}^{2}=0.23 ; \mathrm{Chi}^{2}=23.49, \mathrm{df}=11(\mathrm{P}=0.02) ; \mathrm{I}^{2}=53 \%$

Test for overall effect: $Z=2.61(P=0.009)$

Odds Ratio

dom, $95 \% \mathrm{Cl}$

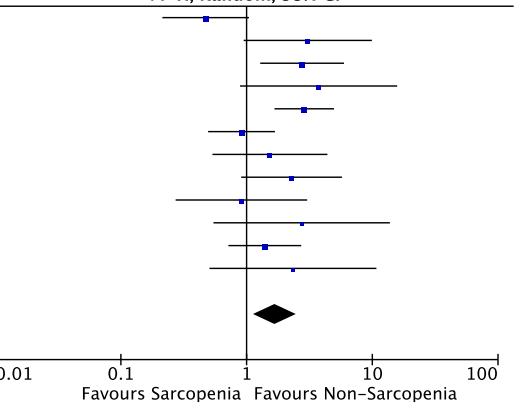

C

Sarcopenia Non-Sarcopenia \begin{tabular}{lrrrrrrr} 
Study or Subgroup & Events & Total & Events & Total & Weight & M-H, Random, 95\% Cl & Year \\
\hline Fukuda 2015 & 3 & 21 & 2 & 78 & $7.4 \%$ & $6.33[0.98,40.74]$ & 2015
\end{tabular} \begin{tabular}{lrrrrrrr}
\hline Fukuda 2015 & 3 & 21 & 2 & 78 & $7.4 \%$ & $6.33[0.98,40.74]$ & 2015 \\
& 5 & 54 & 1 & 239 & $5.8 \%$ & $24.29[2.78,212.48]$ & 2016
\end{tabular} Sato 2016 Wang 2016 Huang 2017 Huang 2017
Kudou 2017 Laou 2017

Zhou 2017

Kawamura 2018 $\begin{aligned} 5.17[1.37,19.44] & 2016 \\ 6.11[3.01,12.40] & 2017\end{aligned}$ $\begin{array}{lll}5.17[1.37,19.44] & 2016 \\ 6.11 & 3.01,12.40] & 2017\end{array}$ $1.28[0.31,5.38] 2017$

$7.83[1.30,47.15] 2017$

$4.10[1.72,9.76] 2017$

Total $(95 \% \mathrm{Cl})$ 422

$840 \quad 18.4 \%$

$1.42[0.62,3.26] 2018$

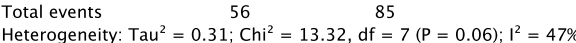

Test for overall effect: $Z=4.64(P<0.00001)$

$4.01[2.23,7.21]$$$
\text { Sarcopenia Non-Sarcopenia Odds Ratio }
$$$$
\text { Odds Ratio }
$$

D Study or Subgroup Fukuda 2015 Wang 2016 Huang 2017 Laou 2017 Yamamoto 2017 Zhou 2017 Kawamura 2018

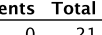

$\begin{array}{rr}1 & \\ 0 & 223 \\ 1 & 391 \\ 1 & 192 \\ 1 & \\ 1 & \\ 26 & 8\end{array}$

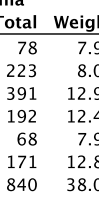

Total $(95 \% \mathrm{Cl})$

348

$1963100.0 \%$

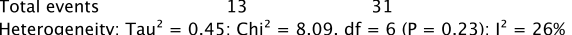

Test for overall effect: $Z=2.82(P=0.005)$

Random, 95\% Cl Year $1.20[0.05,30.56] 2015$ $21.29[0.85,533.95] 2016$ $10.13[0.91,113.11] 2017$ $31.83[2.69,376.46] 2017$ $1.00[0.04,25.43] 2017$ $5.07[0.45,56.90] 2017$ $1.79[0.72,4.45] 2018$

$4.12[1.54,11.02]$

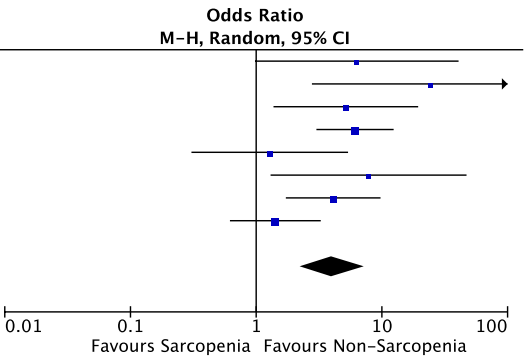

E

\begin{tabular}{ccccccc} 
& \multicolumn{2}{c}{ Sarcopenia } & \multicolumn{2}{c}{ Non-Sarcopenia } & Odds Ratio \\
Study or Subgroup & Events Total & Events & Total & Weight & M-H, Random, $95 \% \mathrm{Cl}$ Year
\end{tabular}

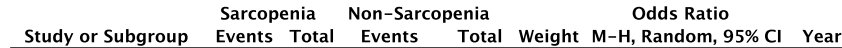
\begin{tabular}{lrrrrrrr} 
Study or Subgroup & Events & Total & Events & Total & Weight & M-H, Random, 95\% Cl & Year \\
\hline Fukuda 2015 & 2 & 21 & 0 & 78 & $5.4 \%$ & $20.13[0.93,436.47]$ & 2015
\end{tabular} Tegels 2015 Wang 2016 Zhuang 2016 Kudou 2017 Laou 2017 Sakurai 2017 Zhou 2017 Huang 2017 Kawamura 2018

$\begin{array}{rr}2 & 21 \\ 7 & 86 \\ 0 & 32 \\ 7 & 389 \\ 0 & 42 \\ 0 & 14 \\ 2 & 14 \\ 0 & 69 \\ 0 & 79 \\ 0 & 111\end{array}$

Total $(95 \% \mathrm{Cl})$

985

Total events 985

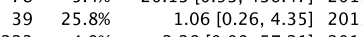
$223 \quad 4.9 \%$ $548 \quad 27.7 \%$ 106 $192 \quad 4.9 \%$ $\begin{array}{lr}1927 & 4.9 \% \\ 17.8 \%\end{array}$ $171 \quad 5.0 \%$ $\begin{array}{ll}391 & 5.0 \% \\ 840 & 5.5 \%\end{array}$ $2.28[0.09,57.21] 2016$ $3.33[0.86,12.95] 2016$ Not estimable 2017 $4.40[0.17,112.95] 2017$ $2.02[0.33,12.21] 2017$ $0.82[0.03,20.32] 2017$ $1.64[0.07,40.56] 2017$ $1.50[0.07,31.53] 2018$

Tau ${ }^{2}=0.00 ; \mathrm{Chi}^{2}=4.01, \mathrm{df}=8(\mathrm{P}=0.86): \mathrm{I}^{2}=0 \%$ Test for overall effect: $Z=2.12(P=0.03)$

\begin{abstract}
$3015100.0 \% \quad 2.17[1.06,4.43]$
\end{abstract}
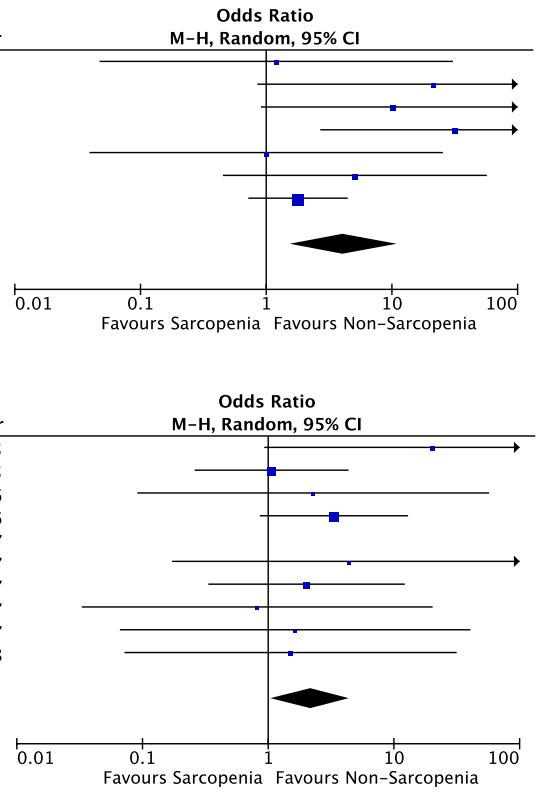
$p<0.001, I^{2} 14 \%$, Fig. 3c). Other factors controlled for by multivariable analysis included age, gender, American Society Anaesthesiology (ASA) Grade, Charlson co-morbidities, diabetes, tumour histology, operation type and American Joint Comission on Cancer stage (T, N, M and overall).

\section{Discussion}

Weight loss and malnutrition remain a central concern for patients at all stages of treatment for gastric cancer. At the time of diagnosis more than half of the patients with gastric cancer present with some degree of weight loss due to underlying tumourigenesis [53]. The inability to maintain body weight is recognised as a poor prognostic factor in sustaining therapeutic response to neoadjuvant or adjuvant treatment and long-term survival [54, 55]. A wide range of approaches have been utilised to assess nutrition in patients with gastric cancers such as anthropometric measures, blood markers, measures of energy expenditure, validated nutritional risk scores, and patient reported dietary history. However, the current review focuses on the assessment of body composition due to the promise that this approach has demonstrated in other areas of medicine and surgery as well as the relative ease with which it might form part of future routine clinical practice [56].

Although four techniques for body composition assessment in patients with gastric cancer have been described, CT and BIA were most commonly used within studies identified in the review. However, none of the studies included in this review discussed the cost of prospectively assessing body composition in patients with gastric cancer. Also, variation in assessment and lack of standardized definition for body composition parameters remain a barrier to adoption into routine clinical practice. DXA is widely considered to be the gold standard non-invasive method of measuring fat mass (FM) and fat free mass (FFM) [57]. The use of DXA is limited in some situations due to the cost of equipment, the need for trained operators, the lack of portability, and the need for exposure to ionizing radiation. $\mathrm{CT}$ also requires expensive equipment and trained operators and exposes the patient to a high dose

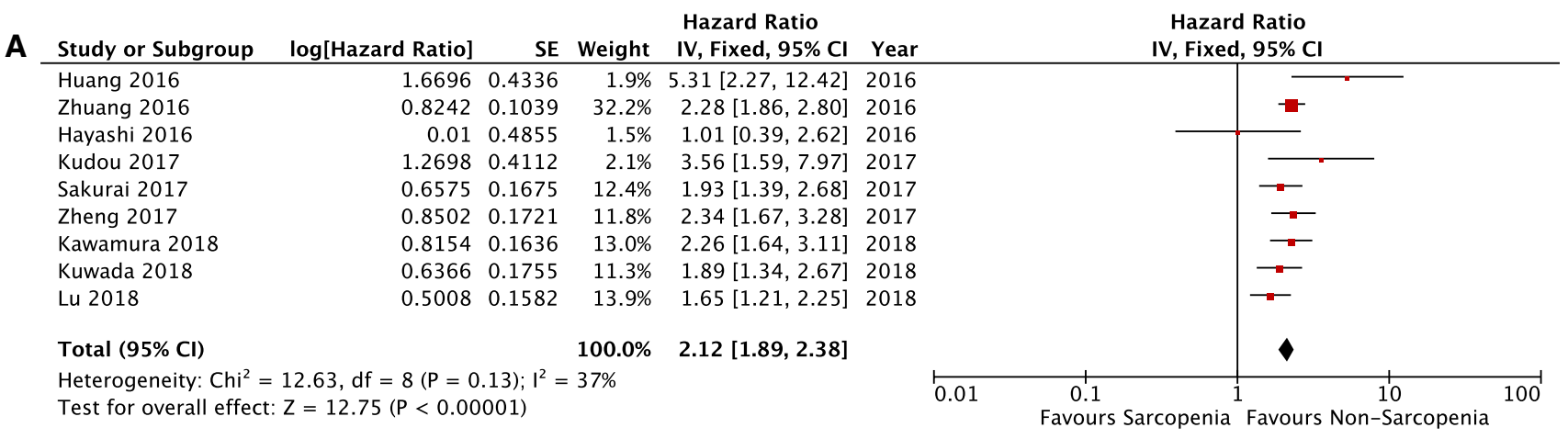

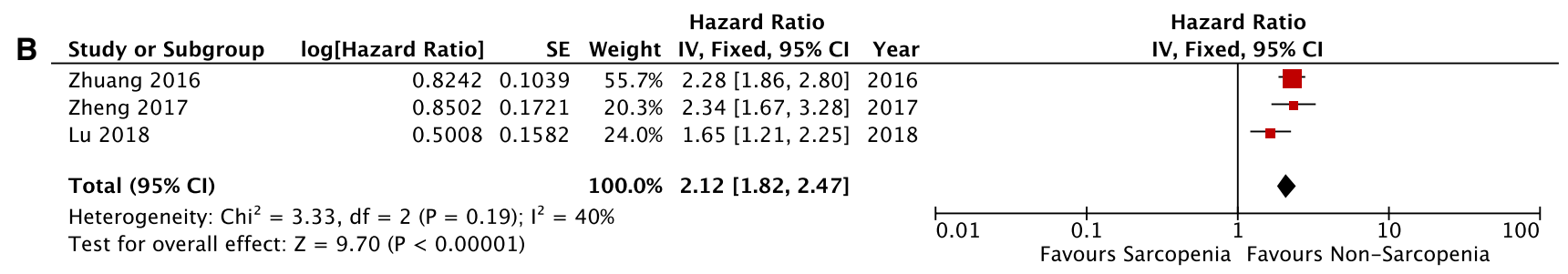

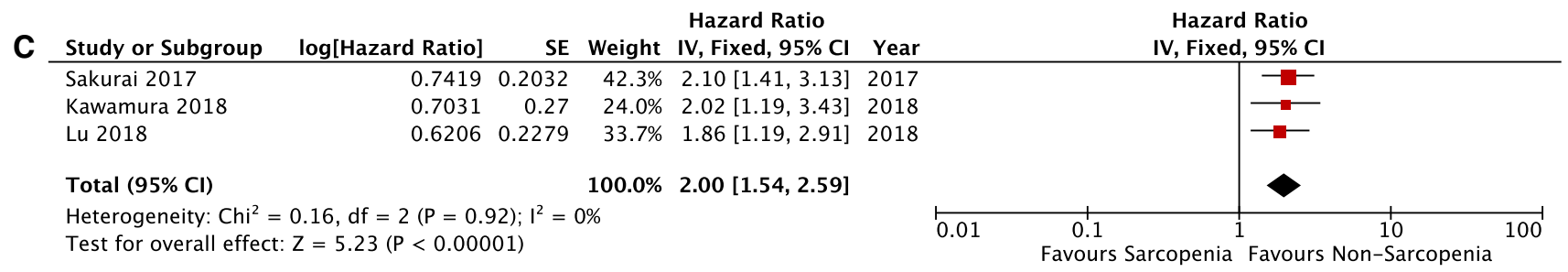

Fig. 3 Summary meta-analysis of studies reporting impact of pre-operative sarcopenia on a overall, b recurrence-free, c cancer-specific survival in patients undergoing gastrectomy 
of radiation. The analysis of body composition from CT necessitates specialist software and training. BIA is practical, non-invasive, and easy to perform. However, studies evaluating BIA have provided inconsistent findings, with some reporting good accuracy [58, 59], whereas others have shown poor results, particularly in obese populations $[60,61]$.

CT scans form a regular part of the standard management of gastric cancer patients and hence offers an opportune method for the assessment of body composition at specific and relevant time-points in the patient treatment pathway. This avoids exposing the patient to repeated imaging and high radiation doses; and it is the authors' recommended method for body composition analysis. A recent study has found excellent correlation between results obtained from DXA and analysis of CT scans [62]. The definition of sarcopenia, a state of severe of depletion of skeletal muscle mass (and function), have been largely established using CT measures and is associated with an increased risk of mortality in many malignant conditions $[63,64]$. This review has highlighted that many studies fail to account for variation introduced by factors such as sex, race, and tumour stage when interpreting and reporting parameters of body composition. Where such controls do not exist it becomes impossible to compare individual studies and to draw meaning that is generalizable.

As sarcopenia is associated with higher rates post-operative morbidity and mortality and poor long-term survival, perioperative interventions are important to reduce these risks. One study in this review reported benefits of oral nutrition supplement to reduce weight loss in the post-operative setting [28]. However, this study did not evaluate its impact on surgical outcomes and long-term survival. Pre-operative rehabilitation is a widely growing concept and adopted into Enhanced Recovery After Surgery (ERAS) protocols across a variety of major surgeries [65]. In gastrectomy for gastric cancer, several studies have reported the improved post-operative outcomes and shorter hospital stay in patients receiving ERAS management as compared to those receiving conventional care [66-68].

The overall strength of conclusions from this review is limited by the heterogeneity of the studies with regards to methods and techniques of assessment of body composition. Subgroup analyses of outcomes, specifically survival, by cancer stage was not possible due to variability in reporting, which is an important confounding factor to note. Furthermore, the absence of studies assessing patients with advanced metastatic gastric cancer who are receiving palliative therapy is also recognized. Finally, a number of other studies were not suitable for inclusion in this review as they reported body composition assessment in a mixed cohort including patients with oesophageal cancer. Collaborative international efforts should now seek to better establish patterns of variation in body composition in gastric cancer patient from disparate racial and geographical backgrounds to establish consensus guidelines. Specifically, the adoption of either a continuous or graded scale for determining sarcopenia and features of adiposity should also be considered.

\section{Conclusion}

This systematic review and meta-analysis have shown higher rates of morbidity and in-hospital mortality following gastrectomy in sarcopenic patients prior to surgery; and an association between sarcopenia and reduced overall, recurrencefree and cancer-specific survival in patients undergoing gastrectomy. Similar findings have been reported for other solid organ tumors [69-71]. This review highlights the need for standardised assessment of body composition as it has the potential to support future decision-making in patients with gastric cancer. It also offers fresh impetus for future studies to further understand underlying mechanisms driving malnutrition with poor outcomes in this patient population. With lack of consensus in regard to optimal methodology and reporting standards, future efforts should be focused at establishing consensus guidelines for body composition assessment in gastric cancer.

Funding None declared.

\section{Compliance with ethical standards}

Conflict of interest The authors declare that they have no conflict of interest.

Ethical standards This study was a systematic review and meta-analysis of all published literature and institutional review deemed this study exempt from full ethical review.

\section{References}

1. Siegel RL, Miller KD, Jemal A. Cancer statistics, 2016. CA Cancer J Clin. 2016;66(1):7-30.

2. Stojcev Z, Matysiak K, Duszewski M, et al. The role of dietary nutrition in stomach cancer. Contemp Oncol (Pozn). 2013;17(4):343-5.

3. Takiguchi S, Takata A, Murakami K, et al. Clinical application of ghrelin administration for gastric cancer patients undergoing gastrectomy. Gastric Cancer. 2014;17(2):200-5.

4. Ebadi M, Martin L, Ghosh S, et al. Subcutaneous adiposity is an independent predictor of mortality in cancer patients. $\mathrm{Br} \mathrm{J}$ Cancer. 2017;117(1):148-55.

5. Palmela C, Velho S, Agostinho L, et al. Body composition as a prognostic factor of neoadjuvant chemotherapy toxicity and outcome in patients with locally advanced gastric cancer. J Gastric Cancer. 2017;17(1):74-87.

6. Rangel EL, Rios-Diaz AJ, Uyeda JW, et al. Sarcopenia increases risk of long-term mortality in elderly patients undergoing 
emergency abdominal surgery. J Trauma Acute Care Surg. 2017;83(6):1179-86.

7. Feliciano EMC, Kroenke CH, Meyerhardt JA, et al. Association of Systemic Inflammation and Sarcopenia With Survival in Nonmetastatic Colorectal Cancer: Results From the C SCANS Study. JAMA Oncol. 2017;3(12):e172319.

8. Liberati A, Altman DG, Tetzlaff J, et al. The PRISMA statement for reporting systematic reviews and meta-analyses of studies that evaluate healthcare interventions: explanation and elaboration. Bmj. 2009;339:b2700.

9. Clavien PA, Barkun J, de Oliveira ML, et al. The Clavien-Dindo classification of surgical complications: five-year experience. Ann Surg. 2009;250(2):187-96.

10. Blum D, Stene GB, Solheim TS, et al. Validation of the Consensus-definition for cancer cachexia and evaluation of a classification model - a study based on data from an international multicentre project (EPCRC-CSA). Ann Oncol. 2014;25(8):1635-42.

11. Fearon K, Strasser F, Anker SD, et al. Definition and classification of cancer cachexia: an international consensus. Lancet Oncol. 2011;12(5):489-95.

12. Stroup DF, Berlin JA, Morton SC, et al. Meta-analysis of observational studies in epidemiology: a proposal for reporting. Metaanalysis Of Observational Studies in Epidemiology (MOOSE) group. JAMA. 2000;283(15):2008-12.

13. Parmar MK, Torri V, Stewart L. Extracting summary statistics to perform meta-analyses of the published literature for survival endpoints. Stat Med. 1998;17(24):2815-34.

14. Aoyama T, Kawabe T, Fujikawa H, et al. Loss of lean body mass as an independent risk factor for continuation of S-1 adjuvant chemotherapy for gastric cancer. Ann Surg Oncol. 2015;22(8):2560-6.

15. Aoyama T, Sato T, Segami K, et al. Risk factors for the loss of lean body mass after gastrectomy for gastric cancer. Ann Surg Oncol. 2016;23(6):1963-70.

16. Aoyama T, Sato T, Hayashi T, et al. Does a laparoscopic approach attenuate the body weight loss and lean body mass loss observed in open distal gastrectomy for gastric cancer? a single-institution exploratory analysis of the JCOG 0912 phase III trial. Gastric Cancer. 2018;21(2):345-52.

17. Chen FF, Zhang FY, Zhou XY, et al. Role of frailty and nutritional status in predicting complications following total gastrectomy with D2 lymphadenectomy in patients with gastric cancer: a prospective study. Langenbecks Arch Surg. 2016;401(6):813-22.

18. Fukuda Y, Yamamoto K, Hirao M, et al. Sarcopenia is associated with severe postoperative complications in elderly gastric cancer patients undergoing gastrectomy. Gastric Cancer. 2016;19(3):986-93.

19. Hayashi N, Ando Y, Gyawali B, et al. Low skeletal muscle density is associated with poor survival in patients who receive chemotherapy for metastatic gastric cancer. Oncol Rep. 2016;35(3):1727-31.

20. Hiki N, Fukunaga T, Yamaguchi T, et al. Increased fat content and body shape have little effect on the accuracy of lymph node retrieval and blood loss in laparoscopic distal gastrectomy for gastric cancer. J Gastrointest Surg. 2009;13(4):626-33.

21. Huang DD, Chen XX, Chen XY, et al. Sarcopenia predicts 1 -year mortality in elderly patients undergoing curative gastrectomy for gastric cancer: a prospective study. J Cancer Res Clin Oncol. 2016;142(11):2347-56.

22. Huang DD, Ji YB, Zhou DL, et al. Effect of surgery-induced acute muscle wasting on postoperative outcomes and quality of life. $\mathrm{J}$ Surg Res. 2017;218:58-66.

23. Huang DD, Zhou CJ, Wang SL, et al. Impact of different sarcopenia stages on the postoperative outcomes after radical gastrectomy for gastric cancer. Surgery. 2017;161(3):680-93.

24. Jeong SH, Lee YJ, Bae K, et al. Clinical factors affecting the length of minilaparotomy incision in laparoscopy-assisted distal gastrectomy. J Laparoendosc Adv Surg Tech A. 2009;19(2):129-33.

25. Kawamura T, Makuuchi R, Tokunaga M, et al. Long-term outcomes of gastric cancer patients with preoperative sarcopenia. Ann Surg Oncol. 2018;25(6):1625-32.

26. Kim JH, Chin HM, Hwang SS, et al. Impact of intra-abdominal fat on surgical outcome and overall survival of patients with gastric cancer. Int J Surg. 2014;12(4):346-52.

27. Kiyama T, Mizutani T, Okuda T, et al. Postoperative changes in body composition after gastrectomy. J Gastrointest Surg. 2005;9(3):313-9.

28. Kobayashi D, Ishigure K, Mochizuki Y, et al. Multi-institutional prospective feasibility study to explore tolerability and efficacy of oral nutritional supplements for patients with gastric cancer undergoing gastrectomy (CCOG1301). Gastric Cancer. 2017;20(4):718-27.

29. Kudou K, Saeki H, Nakashima Y, et al. Prognostic significance of sarcopenia in patients with esophagogastric junction cancer or upper gastric cancer. Ann Surg Oncol. 2017;24(7):1804-10.

30. Kunisaki C, Makino H, Oshima T, et al. Application of the transorally inserted anvil (OrVil) after laparoscopy-assisted total gastrectomy. Surg Endosc. 2011;25(4):1300-5.

31. Kuwada K, Kuroda S, Kikuchi S, et al. Sarcopenia and comorbidity in gastric cancer surgery as a useful combined factor to predict eventual death from other causes. Ann Surg Oncol. 2018;25(5):1160-6.

32. Lee SJ, Kim JY, Ha TK, et al. Changes in lipid indices and body composition one year after laparoscopic gastrectomy: a prospective study. Lipids Health Dis. 2018;17(1):113.

33. Lou N, Chi CH, Chen XD, et al. Sarcopenia in overweight and obese patients is a predictive factor for postoperative complication in gastric cancer: a prospective study. Eur J Surg Oncol. 2017;43(1):188-95.

34. Lu J, Zheng ZF, Li P, et al. A novel preoperative skeletal muscle measure as a predictor of postoperative complications, long-term survival and tumor recurrence for patients with gastric cancer after radical gastrectomy. Ann Surg Oncol. 2018;25(2):439-48.

35. Miyaki A, Imamura K, Kobayashi R, et al. Impact of visceral fat on laparoscopy-assisted distal gastrectomy. Surgeon. 2013;11(2):76-81.

36. Nagata T, Nakase $Y$, Nakamura $K$, et al. Impact of nutritional status on outcomes in laparoscopy-assisted gastrectomy. J Surg Res. 2017;219:78-85.

37. Nishigori T, Tsunoda S, Okabe H, et al. Impact of sarcopenic obesity on surgical site infection after laparoscopic total gastrectomy. Ann Surg Oncol. 2016;23(Suppl 4):524-31.

38. Sakurai K, Kubo N, Tamura T, et al. Adverse effects of low preoperative skeletal muscle mass in patients undergoing gastrectomy for gastric cancer. Ann Surg Oncol. 2017;24(9):2712-9.

39. Sato T, Aoyama T, Hayashi T, et al. Impact of preoperative hand grip strength on morbidity following gastric cancer surgery. Gastric Cancer. 2016;19(3):1008-15.

40. Wang SL, Zhuang CL, Huang DD, et al. Sarcopenia adversely impacts postoperative clinical outcomes following gastrectomy in patients with gastric cancer: a prospective study. Ann Surg Oncol. 2016;23(2):556-64.

41. Yamamoto K, Nagatsuma Y, Fukuda Y, et al. Effectiveness of a preoperative exercise and nutritional support program for elderly sarcopenic patients with gastric cancer. Gastric Cancer. 2017;20(5):913-8.

42. Yamaoka Y, Fujitani K, Tsujinaka T, et al. Skeletal muscle loss after total gastrectomy, exacerbated by adjuvant chemotherapy. Gastric Cancer. 2015;18(2):382-9.

43. Zheng $\mathrm{ZF}$, Lu J, Zheng $\mathrm{CH}$, et al. A novel prognostic scoring system based on preoperative sarcopenia predicts the 
long-term outcome for patients after R0 resection for gastric cancer: experiences of a high-volume center. Ann Surg Oncol. 2017;24(7):1795-803.

44. Zhou CJ, Zhang FM, Zhang FY, et al. Sarcopenia: a new predictor of postoperative complications for elderly gastric cancer patients who underwent radical gastrectomy. J Surg Res. 2017;211:137-46.

45. Zhuang CL, Huang DD, Pang WY, et al. Sarcopenia is an independent predictor of severe postoperative complications and long-term survival after radical gastrectomy for gastric cancer: analysis from a large-scale cohort. Medicine (Baltimore). 2016;95(13):e3164.

46. Naruji Kugimiya EH, Oka K, Kawamura D, Suehiro Y, Takemoto Y, Hamano K. Loss of skeletal muscle mass after curative gastrectomy is a poor prognostic factor. Oncology Letters. 2018;16(1):1341-7.

47. Correia M, Cravo M, Marques-Vidal P, et al. Serum concentrations of TNF-alpha as a surrogate marker for malnutrition and worse quality of life in patients with gastric cancer. Clin Nutr. 2007;26(6):728-35

48. Liedman B, Bosaeus I, Hugosson I, et al. Long-term beneficial effects of a gastric reservoir on weight control after total gastrectomy: a study of potential mechanisms. Br J Surg. 1998;85(4):542-7.

49. Tegels JJ, van Vugt JL, Reisinger KW, et al. Sarcopenia is highly prevalent in patients undergoing surgery for gastric cancer but not associated with worse outcomes. J Surg Oncol. 2015;112(4):403-7.

50. Copland L, Liedman B, Rothenberg E, et al. Effects of nutritional support long time after total gastrectomy. Clin Nutr. 2007;26(5):605-13.

51. Mirkin KA, Luke FE, Gangi A, et al. Sarcopenia related to neoadjuvant chemotherapy and perioperative outcomes in resected gastric cancer: a multi-institutional analysis. J Gastrointest Oncol. 2017;8(3):589-95.

52. Abougergi MS, Travis AC, Saltzman JR. Impact of day of admission on mortality and other outcomes in upper GI hemorrhage: a nationwide analysis. Gastrointest Endosc. 2014;80(2):228-35.

53. Maconi G, Manes G, Porro GB. Role of symptoms in diagnosis and outcome of gastric cancer. World J Gastroenterol. 2008;14(8):1149-55.

54. Correia MI, Waitzberg DL. The impact of malnutrition on morbidity, mortality, length of hospital stay and costs evaluated through a multivariate model analysis. Clin Nutr. 2003;22(3):235-9.

55. Choi WJ, Kim J. Nutritional care of gastric cancer patients with clinical outcomes and complications: a review. Clin Nutr Res. 2016;5(2):65-78.

56. Popuri K, Cobzas D, Esfandiari N, et al. Body composition assessment in axial CT images using FEM-based automatic segmentation of skeletal muscle. IEEE Trans Med Imaging. 2016;35(2):512-20.
57. Lee SY, Gallagher D. Assessment methods in human body composition. Curr Opin Clin Nutr Metab Care. 2008;11(5):566-72.

58. Andreoli A, Melchiorri G, De Lorenzo A, et al. Bioelectrical impedance measures in different position and vs dual-energy X-ray absorptiometry (DXA). J Sports Med Phys Fitness. 2002;42(2):186-9.

59. Jebb SA, Siervo M, Murgatroyd PR, et al. Validity of the leg-toleg bioimpedance to estimate changes in body fat during weight loss and regain in overweight women: a comparison with multicompartment models. Int J Obes (Lond). 2007;31(5):756-62.

60. Deurenberg P. Limitations of the bioelectrical impedance method for the assessment of body fat in severe obesity. Am J Clin Nutr. 1996;64(3 Suppl):449S-452S.

61. Frisard MI, Greenway FL, Delany JP. Comparison of methods to assess body composition changes during a period of weight loss. Obes Res. 2005;13(5):845-54.

62. Tewari N, Awad S, Macdonald IA, et al. A comparison of three methods to assess body composition. Nutrition. 2018;47:1-5.

63. Martin L, Birdsell L, Macdonald N, et al. Cancer cachexia in the age of obesity: skeletal muscle depletion is a powerful prognostic factor, independent of body mass index. J Clin Oncol. 2013;31(12):1539-47.

64. Prado CM, Lieffers JR, McCargar LJ, et al. Prevalence and clinical implications of sarcopenic obesity in patients with solid tumours of the respiratory and gastrointestinal tracts: a population-based study. Lancet Oncol. 2008;9(7):629-35.

65. Ljungqvist $\mathrm{O}$, Scott M, Fearon KC. Enhanced recovery after surgery: a review. JAMA Surg. 2017;152(3):292-8.

66. Ding J, Sun B, Song P, et al. The application of enhanced recovery after surgery (ERAS)/fast-track surgery in gastrectomy for gastric cancer: a systematic review and meta-analysis. Oncotarget. 2017;8(43):75699-711.

67. Mortensen K, Nilsson M, Slim K, et al. Consensus guidelines for enhanced recovery after gastrectomy: Enhanced Recovery After Surgery (ERAS(R)) Society recommendations. Br J Surg. 2014;101(10):1209-29.

68. Sahoo MR, Gowda MS, Kumar AT. Early rehabilitation after surgery program versus conventional care during perioperative period in patients undergoing laparoscopic assisted total gastrectomy. J Minim Access Surg. 2014;10(3):132-8.

69. Levolger S, van Vugt JL, de Bruin RW, et al. Systematic review of sarcopenia in patients operated on for gastrointestinal and hepatopancreatobiliary malignancies. Br J Surg. 2015;102(12):1448-58.

70. Shachar SS, Williams GR, Muss HB, et al. Prognostic value of sarcopenia in adults with solid tumours: A meta-analysis and systematic review. Eur J Cancer. 2016;57:58-67.

71. Boshier PR, Heneghan R, Markar SR, et al. Assessment of body composition and sarcopenia in patients with esophageal cancer: a systematic review and meta-analysis. Dis Esophagus 2018;31:1-11. 